\title{
Efficacy and Safety of a Topical Botanical in Female Androgenetic Alopecia: A Randomized, Single-Blinded, Vehicle-Controlled Study
}

\author{
Alexandros C. Katoulis ${ }^{a}$ Aikaterini l. Liakou ${ }^{a}$ Antigoni Alevizou ${ }^{a}$ \\ Stefanos Bonovas ${ }^{b} \quad$ Evangelia Bozi $^{a} \quad$ Dimitra Kontogiorgi ${ }^{a}$ \\ Dimitrios Rigopoulos ${ }^{\mathrm{a}}$ \\ a 2nd Department of Dermatology and Venereology, National and Kapodistrian University of Athens Medical School,

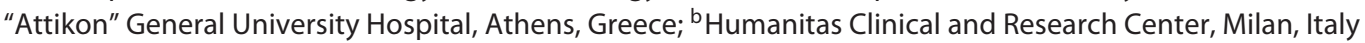

\section{Keywords}

CG210 · Topical · Botanical · Female · Androgenetic alopecia

\begin{abstract}
Introduction: Androgenetic alopecia (AGA) in females is a difficult to treat skin disorder. A novel topical botanical lotion has been approved for its treatment. It acts by increasing BCl-2, perifollicular Langerhans and mast cells, and perifollicular collagen. The aim of this study was to evaluate the efficacy and safety of this lotion in females. Methods: Forty women with AGA were randomized to apply the active lotion or placebo, twice daily for 24 weeks. Subjects were evaluated at 0,12 , and 24 weeks by clinical examination, photographic documentation, quality of life evaluation (DLQI), and trichogram (anagen to telogen ratio). Results: The clinical evaluation demonstrated an increased hair density in the intervention group (great improvement in $7.7 \%$, moderate in $88.5 \%$, and stable in $3.8 \%$ ). The self-assessment score increased from 4.5 to 6.0 (24 weeks), and the DLQI improved from 4 to 3 in the intervention group $(p<0.001)$. The mean anagen to telogen ratio in the intervention group was 2.1, 3.9 , and 6.0 at 0,12 , and 24 weeks, respectively, whereas in
\end{abstract}

\section{KARGER}

(c) 2017 S. Karger AG, Basel

E-Mail karger@karger.com

www.karger.com/sad the control group it was 2.2, 3.8, and 3.3, respectively. Conclusion: The new topical botanical lotion showed remarkable efficacy, with a high degree of patient satisfaction and improvement of their quality of life.

(c) 2017 S. Karger AG, Basel

\section{Introduction}

Androgenetic alopecia (AGA) or pattern hair loss is a noncicatricial alopecia characterized by the gradual conversion of thick, pigmented terminal hairs into vellus hairs through the process of miniaturization. It is an extremely common disorder affecting both genders almost equally [1-4]. AGA appears to be genetically determined, with several genes involved, most notably androgen receptor gene on chromosome $\mathrm{X}$. There are recognized precipitating factors, such as hyperandrogenemia or hyperandrogenism, and possibly microbes, irritants, and UVR, which trigger the disease process. The primary targets may be dermal papilla fibroblasts and the follicular epithelium, with inflammatory cell recruitment and cytokine growth factors and chemokine production in the 
context of follicular microinflammation. These may result in follicular miniaturization, stem cell apoptosis, and perifollicular fibrosis [5-9].

AGA reportedly affects $3-6 \%$ of women aged $<30$ years, increasing to $29-42 \%$ in women aged $\geq 70$ years [10]. The frequency is lower in Asian women compared with those of European descent. There are no published data on the frequency of AGA in African women [11-16]. In females, most often, the frontal hair line is preserved and hair is lost diffusely over the parietal areas and the crown, producing what is called the female pattern hair loss $[17,18]$. The female pattern hair loss occurs less often and to a lesser degree than in men. AGA may produce a considerable cosmetic disfigurement and may have a significant psychosocial impact [19-22].

The only FDA-approved treatment for female AGA is the topical androgen-independent hair growth stimulator minoxidil, in concentrations of 2 and 5\% (FDA, 1992). Other topical treatments include the $5 a$-reductase inhibitor finasteride, topical estrogens, the prostaglandin analogues latanaprost, travoprost, or bimatoprost, and platelet-rich plasma (PRP). Among systematic treatments, $5 a$-reductase inhibitors, such as finasteride and dutasteride, minoxidil, and the androgen receptor antagonists cyproterone acetate and spironolactone are reported to be the most effective. There are also other therapeutic options described, such as laser hair combs, camouflage techniques, and surgical approaches [23-28].

Although much progress has been made, treatment of AGA, especially among females, remains challenging. A novel formula $\left(\mathrm{CG} 210^{\circledR}\right)$ based on onion, lemon, seed, and cocoa extracts has been approved as a treatment option for AGA. The aim of our study was to evaluate the efficacy and safety of this topical botanical lotion in females with AGA.

\section{Materials and Methods}

The design of the study was that of a randomized, single-blinded, vehicle-controlled study. During a 12-month period, 40 unselected women, clinically diagnosed with AGA, were recruited from the Hair Clinic of our Department. All patients were informed about the methods and aims of the study and gave their consent. The study was approved by the Ethics Committee of our hospital. Inclusion criteria were the following: female sex, age $\geq 18$ years, clinical diagnosis of AGA regardless of pattern, and no treatment for AGA or history of other treatment for any reason that might affect the outcome during the previous 12 months. The participants were randomized $(2: 1)$ in a single-blinded manner using simple randomization and were assigned to apply either the active lotion or the vehicle, twice daily, for 24 weeks. During the study period, they were not allowed to use any other active agent for AGA, including food supplements. Subjects were evaluated at 0 , 12 , and 24 weeks by clinical examination, standardized photographic documentation, quality of life evaluation (DLQI), and trichogram. Hair density was assessed by clinical evaluation and comparison of photographs. An improvement of $\leq 25 \%$ was assessed as mild improvement, $>25$ to $\leq 50 \%$ as moderate improvement, and $\geq 50 \%$ as great improvement. For the quality of life assessment, a validated Greek version of the DLQI was employed. For the trichogram, approximately 40 hairs were plugged from the occipital or vertex area of the scalp. The percentage of anagen, catagen, telogen, and dystrophic hairs was recorded. As an assessment tool for the efficacy of the treatment, the anagen to telogen ratio was used. At each visit, the participants were asked to grade their level of satisfaction with the treatment. Possible adverse events were recorded at each visit.

\section{Statistical Analysis}

The ratio of anagen to telogen hair count (anagen to telogen ratio) as well as the quality of life evaluation (DLQI), clinical evaluation, and self-assessment are summarized descriptively as medians and interquartile ranges (IQR) or percentages when appropriate. The primary endpoint of this study is the change of the anagen to telogen ratio from baseline (pre-treatment) to 24 weeks (posttreatment). Secondary endpoints are the change of the anagen to telogen ratio from baseline to 12 weeks as well as the changes in quality of life, clinical evaluation, and self-assessment from baseline to 24 weeks.

Nonparametric tests (the two-sample Wilcoxon rank-sum, the matched-pairs Wilcoxon signed-rank, and the Fisher exact test) that do not require normality were used for the statistical evaluations. Analysis was based on the total number of randomly assigned patients who had both baseline and at least 1 post-treatment measurement (intention-to-treat principle). Probability values of $<0.05$ were considered to be statistically significant. All statistical tests are two-sided. Stata software was used for all analyses (Stata Corp., College Station, TX, USA).

\section{Results}

Forty females with AGA were randomized (2:1) to the intervention and control groups using simple randomization. Of them, 39 patients completed the study (26 assigned to the intervention and 13 to the control group); 1 patient was lost to follow-up. Patients' age ranged from 26 to 81 years (median: 57 years) and disease duration from 1 to 30 years (median: 9 years). At baseline, the two groups were different only in terms of self-assessment; it was worse for patients randomly assigned to the intervention group. The baseline data of the study population, by treatment group, are presented in Table 1.

\section{Clinical Evaluation}

The clinical evaluation demonstrated an increased hair density in the majority of patients receiving the active 
Table 1. Baseline characteristics by treatment group

\begin{tabular}{lccc}
\hline & $\begin{array}{l}\text { Intervention group } \\
(n=26)\end{array}$ & $\begin{array}{l}\text { Control group } \\
(n=13)\end{array}$ & $p$ value \\
\hline Age, years & $57.5(35-67)$ & $47(38-65)$ & 0.71 \\
Disease duration, years & $9.5(6-12)$ & $5(4-15)$ & 0.51 \\
Anagen to telogen ratio at baseline & $2.1(1.7-2.5)$ & $2.2(2.15-2.25)$ & 0.77 \\
DLQI at baseline & $4(2-7)$ & $4(2-5)$ & 0.70 \\
Self-assessment at baseline & $4.5(3-5)$ & $5(5-6)$ & 0.030 \\
Disease classification & & $3(23.1)$ & \\
$\quad$ Olsen pattern & $3(11.5)$ & $3(23.1)$ & \\
$\quad$ Ludwig I & $12(46.2)$ & $7(53.8)$ & 0.073 \\
$\quad$ Ludwig II & $6(23.1)$ & $0(0.0)$ & \\
\hline
\end{tabular}

Data are shown as medians (interquartile ranges) or as $n(\%)$ when appropriate.

Table 2. Summary statistics for pre- and post-treatment anagen to telogen ratio, DLQI, and self-assessment as well as respective changes by treatment group

$\begin{array}{lll}\begin{array}{l}\text { Intervention group } \\ (n=26)\end{array} & \begin{array}{l}\text { Control group } \\ (n=13)\end{array} & \begin{array}{l}\text { Comparison } \\ \text { between groups }\end{array}\end{array}$

\begin{tabular}{|c|c|c|c|}
\hline \multicolumn{4}{|l|}{ Anagen to telogen ratio } \\
\hline At baseline & $2.1(1.7-2.5)$ & $2.2(2.15-2.25)$ & \\
\hline At 3 months & $3.9(2.8-4.3)$ & $3.8(3.8-3.85)$ & \\
\hline Change from baseline to 3 months & $1.7(0.9-2.3) ; p<0.001$ & $1.6(1.6-1.7) ; p=0.001$ & $p=0.78$ \\
\hline At 6 months & $6.0(5.7-6.2)$ & $3.3(3.2-3.4)$ & \\
\hline Change from baseline to 6 months & $3.8(3.2-4.5) ; p<0.001$ & $1.1(1.0-1.2) ; p=0.001$ & $p<0.001$ \\
\hline \multicolumn{4}{|l|}{ DLQI } \\
\hline At baseline & $4(2-7)$ & $4(2-5)$ & \\
\hline At 6 months & $3(0-5)$ & $6(5-7)$ & \\
\hline Change from baseline to 6 months & $-1(-2$ to -1$) ; p<0.001$ & $3(0-4) ; p=0.015$ & $p<0.001$ \\
\hline \multicolumn{4}{|l|}{ Self-assessment } \\
\hline At baseline & $4.5(3-5)$ & $5(5-6)$ & \\
\hline At 6 months & $6(5-8)$ & $5(5-6)$ & \\
\hline Change from baseline to 6 months & $2(1-3) ; p<0.001$ & $0(0-0) ; p=0.56$ & $p<0.001$ \\
\hline
\end{tabular}

Data are shown as medians (interquartile ranges).

lotion (great improvement in 2, moderate improvement in 23 , and stable condition in 1 patient), while all the patients receiving the control lotion were evaluated by the physician as stable ( $p<0.001$ for any improvement) (Fig. 1). No topical or systemic adverse reactions were reported during the study period.

\section{Self-Assessment}

The self-assessment score significantly increased from baseline to 6 months in the intervention group (2, 1-3; $p<0.001)$, but not in the control group $(0,0-0 ; p=0.56)$. Accordingly, the change from baseline was significantly higher in the intervention group $(p<0.001$; Table 2$)$.

\section{Quality of Life}

The quality of life, as assessed with the DLQI, significantly improved in patients of the intervention group $(-1$, -2 to $-1 ; p<0.001)$, while it deteriorated in patients assigned to the control group $(3,0-4 ; p=0.015$; the higher the DLQI score the more quality of life is impaired). Ac- 
cordingly, the change in quality of life (from baseline to 6 months) was in favor of the active treatment $(p<0.001$; Table 2).

\section{Anagen to Telogen Ratio}

At 3 months, the anagen to telogen ratio significantly increased both for the intervention group (median change: 1.7, IQR: $0.9-2.3 ; p<0.001)$ and for the control group $(1.6,1.6-1.7 ; p=0.001)$. The magnitude of this increase did not differ between the two groups $(p=0.78$; Table 2).

At 6 months, the anagen to telogen ratio was again statistically significantly increased from baseline both for the intervention group $(3.8,3.2-4.5 ; p<0.001)$ and the control group $(1.1,1.0-1.2 ; p=0.001)$. This time, however, the change from baseline was significantly higher in the intervention group ( $p<0.001$; Table 2$)$, supporting the hypothesis that the intervention (i.e., botanical lotion) is effective.

\section{Discussion}

According to our results, almost all patients (25 out of 26) clinically improved after receiving the active lotion over a 24 -week period. The anagen to telogen ratio significantly increased from 2.1 at baseline to 3.9 at 12 weeks, and to 6.0 at 24 weeks. The participants in the intervention group assessed themselves as having significantly improved from baseline to 6 months compared to the control group. The quality of life, as assessed with the DLQI, improved in patients of the intervention group, while it worsened in the control group. During the study period, no significant adverse events were reported.

The mechanism of action of this novel formulation appears to be multidimensional. Interestingly, it differs from the mechanism of action of the existing treatment options. It has been found to increase (up to $89.9 \%$ ) Bcl-2, an anti-apoptotic protein that acts during the catagen phase of the hair cycle, hence prolonging the anagen phase and cell survival. Additionally, it downregulates inflammation in the scalp by increasing the number of Langerhans cells and mast cells in the perifollicular area, thus preventing follicular miniaturization and fibrosis

Fig. 1. The photographic documentation of our patients before and after 24-week application of the active lotion shows a clinical improvement.

A Possible New Treatment of Female

Androgenetic Alopecia
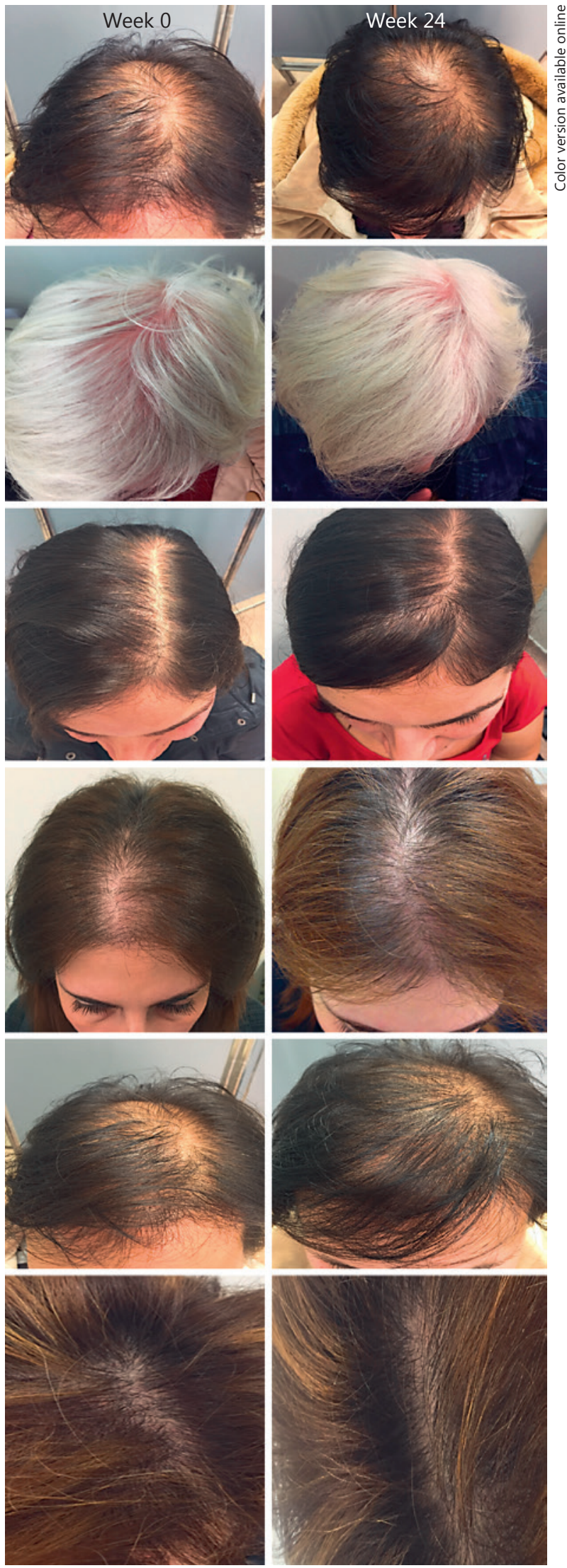

Skin Appendage Disord 2018;4:160-165 DOI: $10.1159 / 000480024$ 
$[29,30]$. Furthermore, it increases (up to 67\%) perifollicular collagen, leading to a better implantation of the hair.

In a recent 12 -week, multicenter, single-arm, open-label study in 56 male subjects with mild to moderate AGA, the safety and efficacy of a twice-daily regimen of 5\% minoxidil foam used in combination with this novel botanical hair solution was evaluated. A significant improvement in scalp hair coverage and perception in as early as 4 weeks $(p<0.001)$ was reported by the investigators. Subject self-ratings were significant for improved hair growth and hair appearance in as few as 4 weeks $(p<$ $0.05)$. The regimen was well tolerated, and subjects indicated a high degree of satisfaction [29].

Another multicenter, single-arm, open-label study in 54 female subjects evaluated the safety and efficacy of a twice-daily regimen of $2 \%$ minoxidil solution used in combination with the new botanical hair solution for 12 weeks. Investigator ratings indicated significant improvement in hair growth and overall treatment benefits in as early as 6 weeks $(p<0.001)$. Subject self-ratings indicated significant satisfaction with hair volume and quality improvement at week $6(p<0.001)$. Subjects also indicated an increase in self-confidence and attractiveness at week $12(p<0.001)[30]$.

A 12-month, randomized, double-blind, placebo-controlled trial using CG210 (the novel topical anti-hair loss product) in $20 \mathrm{AGA}$ volunteers already using finasteride $1 \mathrm{mg}$ for at least 3 years was also published. Hair diameters were assessed and compared for hair pattern improvement. The increase of hair diameter in the "finasteride $1 \mathrm{mg}$ + topical CG210" group was $37.7 \%$ more than that in the "finasteride $1 \mathrm{mg}+$ topical placebo" group $(p=0.002)$. No side effects were observed [31].

In another open prospective study of 20 male patients with AGA, immunohistochemical analyses of scalp biopsies showed a significant increase in the cutaneous immune response after treatment: $73.9 \%$ increase in CD1A+
Langerhans cells ( $p=0.003$, paired $t$ test), $41.7 \%$ increase in the cellular proliferation marker Ki-67+ $(p=0.012)$, and $89 \%$ increase in BCL-2+ anti apoptotic proteins $(p=$ $001)$. The product was also found to be tolerable and safe [32].

Our results are in agreement with the already published data concerning this new botanical lotion. In addition, we demonstrated an improvement of AGA in females, as indicated by the progressive increase of the anagen to telogen ratio in the trichogram. The anagen to telogen ratio is considered to be a valid indicator of the follicle activity and its restoration to normal appears to be a strong documentation of the efficacy of this new botanical. CG210 seems to be a novel, promising treatment of female AGA. It acts with an original triple mechanism, different from the already existing therapies. It is very safe. In our study, it showed clinical and laboratorial (trichogram) efficacy, with a high degree of satisfaction and improvement of patients' quality of life. The results were evident in 3 months and significantly different after 6 months compared to the control group. Long-term follow-up of the patients will clarify the duration of the result achieved. In conclusion, CG210 appears to be an effective and safe alternative to minoxidil topical treatment for AGA in females. Future studies, including comparison with minoxidil, may support its use as a first-line choice in the treatment of AGA.

\section{Statement of Ethics}

All patients were informed about the methods and aims of the study and gave their consent. The study was approved by the Ethics Committee of our hospital.

\section{Disclosure Statement}

The authors report no conflict of interest.

\section{References}

1 Rogers NE, Avram MR: Medical treatments for male and female pattern hair loss. J Am Acad Dermatol 2008;59:547-566.

2 Blume-Peytavi U, et al: S1 guideline for diagnostic evaluation in androgenetic alopecia in men, women and adolescents. Br J Dermatol 2011;164:5-15.
3 Blumeyer A, et al: Evidence-based (S3) guideline for the treatment of androgenetic alopecia in women and in men. J Dtsch Dermatol Ges 2011;9:1-57.

4 Norwood O, Lehr B: Female androgenetic alopecia: a separate entity. Dermatol Surg 2000; 26:679-682.

5 Trüeb RM: Molecular mechanisms of androgenetic alopecia. Exp Gerontol 2002;37:981990.
6 Olsen EA: Female pattern hair loss. J Am Acad Dermatol 2002;45:70-80.

7 Tosti A, Piraccini BM: Androgenetic alopecia; in Tosti A, Piraccini BM (eds): Diagnosis and Treatment of Hair Disorders: An Evidence Based Atlas. London, Taylor and Francis, 2006, pp 45-56.
Katoulis/Liakou/Alevizou/Bonovas/Bozi/ Kontogiorgi/Rigopoulos 
8 Yip L, Rufaut N, Sinclair R: Role of genetics and sex steroid hormones in male androgenetic alopecia and female pattern hair loss: an update of what we now know. Australas J Dermatol 2011;52:81-88.

9 Arias-Santiago S, et al: Androgenetic alopecia and cardiovascular risk factors in men and women: a comparative study. J Am Acad Dermatol 2010;63:420-429.

10 Norwood OT: Incidence of female androgenetic alopecia (female pattern alopecia). Dermatol Surg 2001;27:53-54.

11 Birch MP, Messenger JF, Messenger AG: Hair density, hair diameter and the prevalence of female pattern hair loss. Br J Dermatol 2001; 144:297-304.

12 Paik JH, Yoon JB, Sim WY, et al: The prevalence and types of androgenetic alopecia in Korean men and women. Br J Dermatol 2001; 145:95-99.

13 Price VH: Androgenetic alopecia in women. J Investig Dermatol Symp Proc 2003;8:24-27.

14 Gan DCC, Sinclair RD: Prevalence of male and female pattern hair loss in Maryborough. J Investig Dermatol Symp Proc 2005;10:184189.

$15 \mathrm{Xu}$ F, Sheng YY, Mu Zl, et al: Prevalence and types of androgenetic alopecia in Shanghai, China: a community-based study. Br J Dermatol 2009;160:629-632.

16 O'Tar T: Incidence of female androgenetic alopecia (female pattern alopecia). Dermatol Surg 2001;27:53-54.
17 Lee AT, Zane LT: Dermatologic manifestations of polycystic ovary syndrome. Am J Clin Dermatol 2007;8:201-219.

18 Bergfeld WF: Androgenetic alopecia: an autosomal dominant disorder. Am J Med 1995;98: 95-98.

19 Cash TF: The psychological effects of androgenetic alopecia in men. J Am Acad Dermatol 1992;26:926-931.

20 Donk J, et al: Psychological characteristics of women with androgenetic alopecia: a controlled study. Br J Dermatol 1991;125:248252.

21 Venning VA, Dawber RPR: Patterned androgenic alopecia in women. J Am Acad Dermatol 1988;18:1073-1077.

22 Cash TF, Price VH, Savin RC: Psychological effects of androgenetic alopecia on women: comparisons with balding men and with female control subjects. J Am Acad Dermatol 1993;29:568-575.

23 Olsen EA, Messenger AG, Shapiro J, et al: Evaluation and treatment of male and female pattern hair loss. J Am Acad Dermatol 2005; 52:301-311.

24 DeVillez RL, et al: Androgenetic alopecia in the female: treatment with $2 \%$ topical minoxidil solution. Arch Dermatol 1994;130:303307.

25 Price VH, et al: Lack of efficacy of finasteride in postmenopausal women with androgenetic alopecia. J Am Acad Dermatol 2000;43:768776.
26 Vexiau P, et al: Effects of minoxidil 2\% versus cyproterone acetate treatment on female androgenetic alopecia: a controlled, 12-month randomized trial. Br J Dermatol 2002;146: 992-999.

27 Whiting DA, Jacobson C: Treatment of female androgenetic alopecia with minoxidil 2\%. Int J Dermatol 1992;31:800-804.

28 Blume-Peytavi $U$, et al: A randomized, singleblind trial of $5 \%$ minoxidil foam once daily versus $2 \%$ minoxidil solution twice daily in the treatment of androgenetic alopecia in women. J Am Acad Dermatol 2011;65:11261134.

29 Keaney TC, Pham H, von Grote E, Meckfessel MH: Efficacy and safety of minoxidil $5 \%$ foam in combination with a botanical hair solution in men with androgenic alopecia. J Drugs Dermatol 2016;15:406-412.

30 McMichael A, Pham A, von Grote E, Meckfessel MH: Efficacy and safety of minoxidil 2\% solution in combination with a botanical hair solution in women with female pattern hair loss/androgenic alopecia. J Drugs Dermatol 2016;15:398-404.

31 Takeda A, Sato A, Zhang L, Harti S, Cauwenbergh G, et al: CG210 enables finasteride $1 \mathrm{mg}$ users to further improve hair pattern: a randomized, double-blind, placebo-controlled pilot study. Hair Ther Transplant 2013;3:107.

32 Cucé LC, Rodrigues CJ, Patriota RCR: Celli$u^{\circledR}$ GC: evaluation of a new natural active ingredient in $210 \mathrm{mg} / \mathrm{mL}$ topical solution, through scalp biopsy. Surg Cosmet Dermatol 2011;3:123-128. 